\title{
IMPACT OF PHYSICOCHEMICAL CHARACTERISTICS OF WATER ON COEFFICIENT OF PIPE FRICTION
}

\author{
A.G Gaddamwar ${ }^{1}$, K.R.Banarse ${ }^{2}$, S.P.Yawale ${ }^{3}$, P.R.Rajput ${ }^{4}$ \\ ${ }^{1}$ Head of Department of Engineering Chemistry, DES's College of Engineering and Technology, SGB Amravati \\ University, Amravati, Maharashtra, India \\ ${ }^{2}$ Head of Department of Engineering Physics, DES's College of Engineering and Technology, SGB Amravati \\ University, Amravati, Maharashtra, India \\ ${ }^{3}$ Associate Professor, Department of Physics, Govt. Vidharbha Institute of Science and Humanities SGBAU Amravati \\ University, Amravati, Maharashtra, India \\ ${ }^{4}$ Associate Professor, Department of Chemistry, Vidyabharti Science College, Amravati
}

\begin{abstract}
Frictions are of two type i.e. internal friction and external friction, internal friction as well as external friction of water mainly depends upon Physico-chemical characteristic of water (liquids). It is due to turbidity, hardness, conductivity, temperature and dissolved oxygen of water. External friction can be reduced by different techniques such as by internal coating of pipes in which liquid flow and by increasing or decreasing diameter of pipes. Still it has some internal and external frictional losses. Those frictional losses can also be reduced by the addition quantitative and qualitative amount of surfactant and other chemicals in the water samples. Co-efficient of Friction ' $F$ ' of pipe play very important role in fluid mechanics. Co-efficient of Friction initially decreases by the addition of sodium hydroxide and surfactant at diameter two but at diameter one $F$ value initially decreases then increases.
\end{abstract}

Keywords: Analysis of water, Friction, Co-efficient of Friction, hardness, surfactant

\section{INTRODUCTION}

The friction factor ' $\mathrm{f}$ ' depends on the velocity of flow, the pipe diameter, the fluid density and viscosity and the roughness of the pipe, Physico-chemical quality of water. The resistance of flowing fluid is known as frictional resistance produced due to directional flow which depends upon the type of flow of liquid in a pipe line. It may be laminar. Following laws which obey the frictional resistance are 1.Directly proportional to velocity of flowing fluid. 2 . Independent of pressure 3.Proportional to area of constant surface 4. Affected by a variation of flowing fluid, The occurrence of laminar or turbulent flow as generated by realistic magnitude of inertia of viscous fluid show that velocity of flow even true liquid having very small velocity frictional resistance in direction of fluid flow and different flow reduced the pressure intensity and difference of pressure reading between any two section is equal to loss of head due to friction. In the formula, hf is coefficient of friction, $\mathrm{f}$ is frictional resistance, $\mathrm{L}$ is the distance between two manometer readings, $\mathrm{v}$ is velocity of fluid, $\mathrm{g}$ is acceleration due to gravity, $\mathrm{d}$ is diameter of pipe. Reynolds number of flow of fluid to the aperture is generated by means of a regulating valve provided at the end of each pipe as shown in figure, a common inlet valve is provided in main supply line. For the whole apparatus pipe may be 2 or 3 centimeter apart. Sufficient length of pipe line is provided between various fittings.

\section{MATERIALS AND METHOD}

\subsection{Investigation of Co-efficient of Pipe Friction}

The manometer rubber tubing is connected to guage points of one of the pipe line. The inlet value is open by keeping the outlet valve close. Now the outlet valve is opened partially keeping the inlet valve fully open. The flow is allowed to get established and then manometer readings are taken. The actual discharge by collecting the water into the tank is measured. Above steps are repeated for four different discharges by taking different samples, and also repeated for other pipes having different diameters.

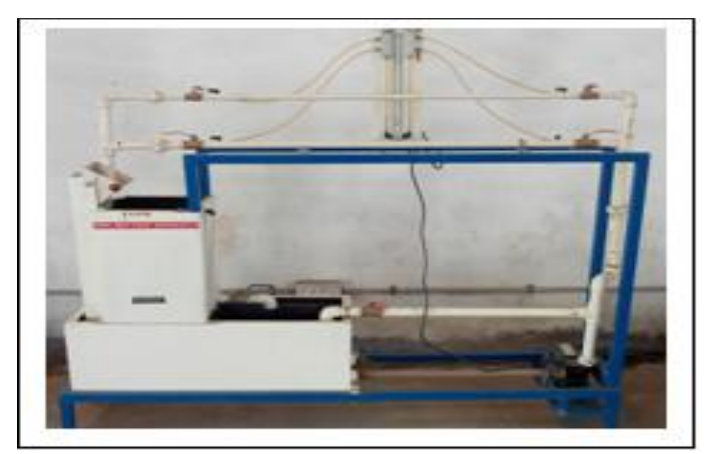

Fig-1 Pipe Friction Apparatus to measure co-efficient of Friction

\subsection{Investigation of $\mathrm{pH}$}

The $\mathrm{pH}$ of water is investigated with the help of Digital $\mathrm{pH}$ meter after calibration of instrument at room temperature. 


\subsection{Investigation of TDS}

The TDS of water bodies investigated with the help of Digital TDS meter at $350 \mathrm{c}$.

\subsection{Investigation of Conductivity}

The conductivity of water bodies are investigated with the help of Digital conductivity meter after calibration of instrument using 0.01 molar $\mathrm{KCl}$ solutions.

\subsection{Investigation of Hardness}

The hardness of water sample is investigated by using EDTA complex metric titration method.

\subsection{Investigation of Dissolved Oxygen}

The oxygen of water sample is investigated by using chemical method. Important parameters of water bodies are given in the Table No1.

Table-1 Co-efficient of Pipe Friction with Bore well Water sample

\begin{tabular}{|c|c|c|c|c|c|c|c|c|c|c|}
\hline \multirow{2}{*}{$\begin{array}{l}\text { Sr. } \\
\text { No. }\end{array}$} & \multirow{2}{*}{$\begin{array}{l}\text { Diameter } \\
\text { of Pipe } \\
\text { 'd' (m) }\end{array}$} & \multirow{2}{*}{$\begin{array}{l}\text { Area of } \\
\text { Tank } \\
\left(\mathrm{m}^{2}\right)\end{array}$} & \multirow{2}{*}{$\begin{array}{l}\text { Height of } \\
\text { Water } \\
\text { Collected } \\
(\mathrm{m})\end{array}$} & \multirow{2}{*}{$\begin{array}{l}\text { Time } \\
\text { 't' } \\
(\mathrm{sec})\end{array}$} & \multirow{2}{*}{$\begin{array}{l}\text { Actual } \\
\text { Discharg } \\
\mathrm{e} \\
\mathrm{Q}=\mathrm{V} / \mathrm{t}\end{array}$} & \multicolumn{2}{|c|}{ Piezometer Reading } & \multirow{2}{*}{$\begin{array}{l}\text { Head loss } \\
\text { due to } \\
\text { friction } \\
\mathrm{h}_{\mathrm{f}}=\mathrm{h}_{1} \sim \mathrm{h}_{2}\end{array}$} & \multirow{2}{*}{$\begin{array}{l}\mathrm{F} \\
\frac{2 h_{f} g d}{4 L v^{2}}\end{array}=$} & \multirow{2}{*}{$\begin{array}{l}\text { Mean } \\
\text { F }\end{array}$} \\
\hline & & & & & & $\mathrm{h}_{1}(\mathrm{~m})$ & $\mathrm{h}_{2}(\mathrm{~m})$ & & & \\
\hline 1 & $2 \times 10^{-2}$ & $\begin{array}{l}900 \times \\
10^{-4}\end{array}$ & $15 \times 10^{-2}$ & 103 & $\begin{array}{ll}1.31 & \times \\
10^{-4} & \\
\end{array}$ & $8 \times 10^{-2}$ & $6.7 \times 10^{-2}$ & $1.3 \times 10^{-2}$ & $\begin{array}{l}6.62 \\
10^{-3} \\
\end{array}$ & \multirow{3}{*}{$\begin{array}{l}5.77 \times \\
10^{-3}\end{array}$} \\
\hline 2 & $2 \times 10^{-2}$ & $\begin{array}{l}900 \times \\
10^{-4}\end{array}$ & $15 \times 10^{-2}$ & 84 & $\begin{array}{l}1.60 \times \\
10^{-4}\end{array}$ & $8.7 \times 10^{-2}$ & $7 \times 10^{-2}$ & $1.7 \times 10^{-2}$ & $5.6 \times 10^{-}$ & \\
\hline 3 & $2 \times 10^{-2}$ & $\begin{array}{ll}900 & \times \\
10^{-4} & \\
\end{array}$ & $15 \times 10^{-2}$ & 62 & $\begin{array}{ll}2.17 & \times \\
10^{-4} & \\
\end{array}$ & $9.4 \times 10^{-2}$ & $6.6 \times 10^{-2}$ & $2.8 \times 10^{-2}$ & $\begin{array}{l}5.10 \times \\
10^{-3}\end{array}$ & \\
\hline 4 & $1.5 \times 10^{-2}$ & $\begin{array}{ll}900 & \times \\
10^{-4} & \\
\end{array}$ & $15 \times 10^{-2}$ & 169 & $7.9 \times 10^{-4}$ & $8.7 \times 10^{-2}$ & $8.3 \times 10^{-2}$ & $0.4 \times 10^{-2}$ & $\begin{array}{l}4.15 \\
10^{-3} \\
\end{array}$ & \multirow{3}{*}{$\begin{array}{l}2.524 \\
\times 10^{-3}\end{array}$} \\
\hline 5 & $1.5 \times 10^{-2}$ & $\begin{array}{l}900 \times \\
10^{-4}\end{array}$ & $15 \times 10^{-2}$ & 63 & $\begin{array}{l}2.14 \\
10^{-4}\end{array}$ & $9.6 \times 10^{-2}$ & $7.8 \times 10^{-2}$ & $1.8 \times 10^{-2}$ & $\begin{array}{l}2.54 \\
10^{-3}\end{array}$ & \\
\hline 6 & $1.5 \times 10^{-2}$ & $\begin{array}{l}900 \times \\
10^{-4}\end{array}$ & $15 \times 10^{-2}$ & 33 & $\begin{array}{l}4.09 \times \\
10^{-4}\end{array}$ & $9.7 \times 10^{-2}$ & $7.6 \times 10^{-2}$ & $2.27 \times 10^{-2}$ & $\begin{array}{l}8.82 \\
10^{-4}\end{array}$ & \\
\hline
\end{tabular}

Table-2 Co-efficient of Pipe Friction with Bore well Watersample+0.05M NaOH solution:

\begin{tabular}{|c|c|c|c|c|c|c|c|c|c|c|}
\hline \multirow{2}{*}{$\begin{array}{l}\text { Sr. } \\
\text { No. }\end{array}$} & \multirow{2}{*}{$\begin{array}{l}\text { Diameter } \\
\text { of Pipe } \\
\text { 'd' (m) }\end{array}$} & \multirow{2}{*}{$\begin{array}{l}\text { Area } \\
\text { of } \\
\text { Tank } \\
\left(\mathrm{m}^{2}\right) \\
\end{array}$} & \multirow{2}{*}{$\begin{array}{l}\text { Height of } \\
\text { Water } \\
\text { Collected } \\
\text { (m) }\end{array}$} & \multirow{2}{*}{$\begin{array}{l}\text { Time } \\
\text { ' } t \text { ' } \\
(\mathrm{sec})\end{array}$} & \multirow{2}{*}{$\begin{array}{l}\text { Actual } \\
\text { Discharge } \\
Q=V / t\end{array}$} & \multicolumn{2}{|c|}{ Piezometer Reading } & \multirow{2}{*}{$\begin{array}{l}\text { Head loss } \\
\text { due to } \\
\text { friction } \\
h_{\mathrm{f}}=\mathrm{h}_{1} \sim \mathrm{h}_{2}\end{array}$} & \multirow{2}{*}{$\begin{array}{l}\mathrm{F}= \\
\frac{2 h_{f} g d}{4 L v^{2}}\end{array}$} & \multirow{2}{*}{$\begin{array}{l}\text { Mean } \\
\mathrm{F}\end{array}$} \\
\hline & & & & & & $\mathrm{h}_{1}(\mathrm{~m})$ & $\mathrm{h}_{2}(\mathrm{~m})$ & & & \\
\hline 1 & $2 \times 10^{-2}$ & $\begin{array}{l}900 \times \\
10^{-4}\end{array}$ & $15 \times 10^{-2}$ & 77 & $1.75 \times 10^{-4}$ & $7.7 \times 10^{-2}$ & $9.5 \times 10^{-2}$ & $1.8 \times 10^{-2}$ & $\begin{array}{l}5.06 \\
\times 10^{-3} \\
\end{array}$ & \multirow{2}{*}{$\begin{array}{l}3.76 \\
\times 10^{-3}\end{array}$} \\
\hline 2 & $2 \times 10^{-2}$ & $\begin{array}{l}900 \times \\
10^{-4}\end{array}$ & $15 \times 10^{-2}$ & 57 & $2.36 \times 10^{-4}$ & $7.6 \times 10^{-2}$ & $9.2 \times 10^{-2}$ & $1.6 \times 10^{-2}$ & $\begin{array}{l}2.46 \\
\times 10^{-3} \\
\end{array}$ & \\
\hline 3 & $1.5 \times 10^{-2}$ & $\begin{array}{l}900 \times \\
10^{-4}\end{array}$ & $15 \times 10^{-2}$ & 128 & $1.05 \times 10^{-4}$ & $8.7 \times 10^{-2}$ & $8.3 \times 10^{-2}$ & $0.5 \times 10^{-2}$ & $\begin{array}{l}2.91 \\
\times 10^{-3}\end{array}$ & \multirow{2}{*}{$\begin{array}{l}2.02 \\
\times 10^{-3}\end{array}$} \\
\hline 4 & $1.5 \times 10^{-2}$ & $\begin{array}{l}900 \times \\
10^{-4}\end{array}$ & $15 \times 10^{-2}$ & 46 & $2.93 \times 10^{-4}$ & $9.2 \times 10^{-2}$ & $7.7 \times 10^{-2}$ & $1.5 \times 10^{-2}$ & $\begin{array}{l}1.13 \\
\times 10^{-3}\end{array}$ & \\
\hline
\end{tabular}

Table-3 Co-efficient of Pipe Friction with bore well Water sample $+0.05 \mathrm{M} \mathrm{NaOH}$ solution + surfactant 13 gm

\begin{tabular}{|c|c|c|c|c|c|c|c|c|c|c|}
\hline \multirow{2}{*}{$\begin{array}{l}\text { Sr. } \\
\text { No. }\end{array}$} & \multirow{2}{*}{$\begin{array}{l}\text { Diameter } \\
\text { of Pipe } \\
\text { 'd' }(m)\end{array}$} & \multirow{2}{*}{$\begin{array}{l}\text { Area of } \\
\text { Tank } \\
\left(\mathrm{m}^{2}\right)\end{array}$} & \multirow{2}{*}{$\begin{array}{l}\text { Height of } \\
\text { Water } \\
\text { Collected } \\
\text { (m) }\end{array}$} & \multirow{2}{*}{$\begin{array}{l}\text { Time } \\
\text { ' } \mathrm{t} \text { ' } \\
(\mathrm{sec})\end{array}$} & \multirow{2}{*}{$\begin{array}{l}\text { Actual } \\
\text { Discharge } \\
Q=V / t\end{array}$} & \multicolumn{2}{|c|}{ Piezometer Reading } & \multirow{2}{*}{$\begin{array}{l}\text { Head loss } \\
\text { due to } \\
\text { friction } \\
h_{\mathrm{f}}=\mathrm{h}_{1} \sim \mathrm{h}_{2}\end{array}$} & \multirow{2}{*}{$\begin{array}{l}\mathrm{F} \\
\frac{2 h_{f} g d}{4 L v^{2}}\end{array}=$} & \multirow{2}{*}{$\begin{array}{l}\text { Mean } \\
\text { F }\end{array}$} \\
\hline & & & & & & $\mathrm{h}_{1}(\mathrm{~m})$ & $\mathrm{h}_{2}(\mathrm{~m})$ & & & \\
\hline 1 & $2 \times 10^{-2}$ & $\begin{array}{l}900 \times \\
10^{-4}\end{array}$ & $15 \times 10^{-2}$ & 80 & $1.68 \times 10^{-4}$ & $7.9 \times 10^{-2}$ & $9.1 \times 10^{-2}$ & $1.2 \times 10^{-2}$ & $\begin{array}{l}3.64 \times \\
10^{-3}\end{array}$ & \multirow{2}{*}{$\begin{array}{l}2.45 \times \\
10^{-3}\end{array}$} \\
\hline 2 & $2 \times 10^{-2}$ & $\begin{array}{l}900 \\
10^{-4} \\
\end{array}$ & $15 \times 10^{-2}$ & 57 & $2.36 \times 10^{-4}$ & $7.8 \times 10^{-2}$ & $9.0 \times 10^{-2}$ & $1.2 \times 10^{-2}$ & $\begin{array}{l}1.84 \times \\
10^{-3}\end{array}$ & \\
\hline 3 & $1.5 \times 10^{-2}$ & $\begin{array}{l}900 \\
10^{-4}\end{array}$ & $15 \times 10^{-2}$ & 129 & ${ }_{4}^{1.046 \times 10^{-}}$ & $8.1 \times 10^{-2}$ & $8.7 \times 10^{-2}$ & $0.6 \times 10^{-2}$ & $\begin{array}{l}3.55 \times \\
10^{-3}\end{array}$ & \multirow{2}{*}{$\begin{array}{l}2.40 \times \\
10^{-3}\end{array}$} \\
\hline 4 & $1.5 \times 10^{-2}$ & $\begin{array}{l}900 \\
10^{-4}\end{array}$ & $15 \times 10^{-2}$ & 77 & $1.75 \times 10^{-4}$ & $8.2 \times 10^{-2}$ & $8.8 \times 10^{-2}$ & $0.6 \times 10^{-2}$ & $\begin{array}{l}1.26 \times \\
10^{-3}\end{array}$ & \\
\hline
\end{tabular}


Table-4 Co-efficient of Pipe Friction with bore well Water sample $+0.05 \mathrm{M} \mathrm{NaOH}$ solution + surfactant 39 gm:

\begin{tabular}{|l|l|l|l|}
\hline $\begin{array}{l}\text { Sr. } \\
\text { No. }\end{array}$ & Particulars of Sample & $\begin{array}{l}\text { Co-efficient of Friction 'F' } \\
\text { (diameter of pipe }=2 \mathrm{~cm})\end{array}$ & $\begin{array}{l}\text { Co-efficient of Friction 'F' } \\
(\text { diameter of pipe }=1.5 \\
\mathrm{cm})\end{array}$ \\
\hline 1 & Sample No. 1(Bore well Water) & $5.77 \times 10^{-3}$ & $2.524 \times 10^{-3}$ \\
\hline 2 & Sample No. 2 (Bore well Water $+0.05 \mathrm{M} \mathrm{NaOH})$ & $2.45 \times 10^{-3}$ & $2.40 \times 10^{-3}$ \\
\hline 3 & $\begin{array}{l}\text { Sample No. 3 (Bore well Water }+0.05 \mathrm{M} \mathrm{NaOH} \text { solution }+ \\
\text { surfactant 13 gm })\end{array}$ & $3.76 \times 10^{-3}$ & $2.02 \times 10^{-3}$ \\
\hline 4 & $\begin{array}{l}\text { Sample No. 4 (Bore well Water sample }+0.05 \mathrm{M} \mathrm{NaOH} \\
\text { solution }+ \text { surfactant 39 gm) }\end{array}$ & $3.1 \times 10^{-3}$ & $5.61 \times 10^{-3}$ \\
\hline 5 & $\begin{array}{l}\text { Remark } \\
\text { F value decreases from top to } \\
\text { bottom }\end{array}$ & $\begin{array}{l}\text { F value initially decreases } \\
\text { then increases }\end{array}$ \\
\hline
\end{tabular}

Table-5 Comparison of Co-efficient of Friction of different samples

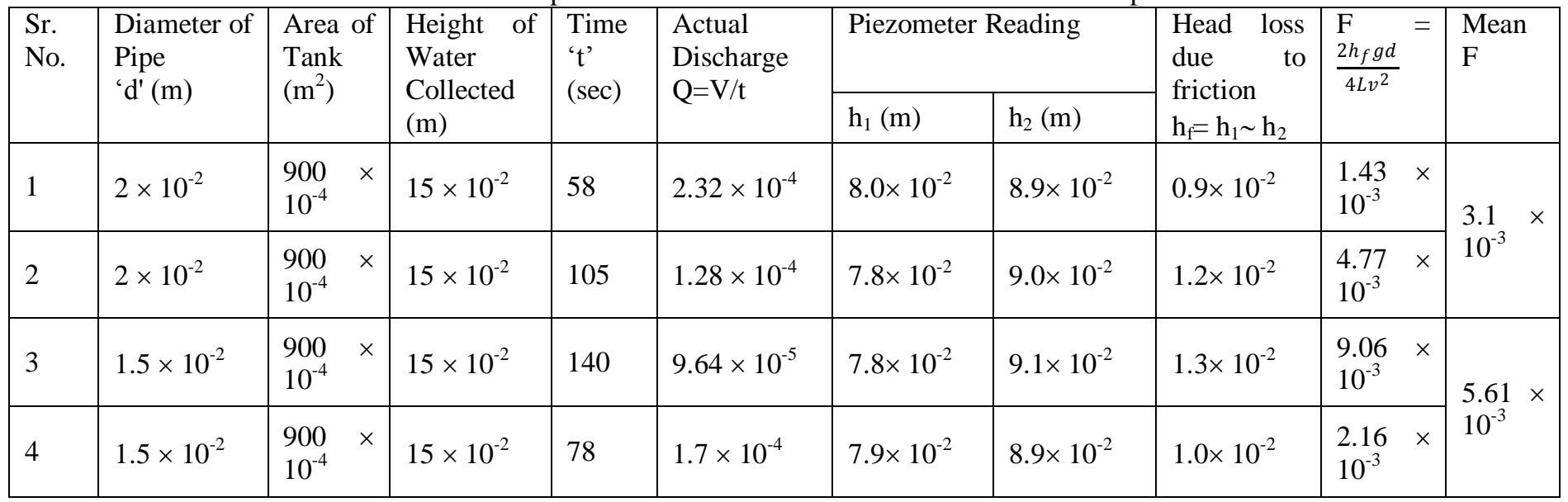

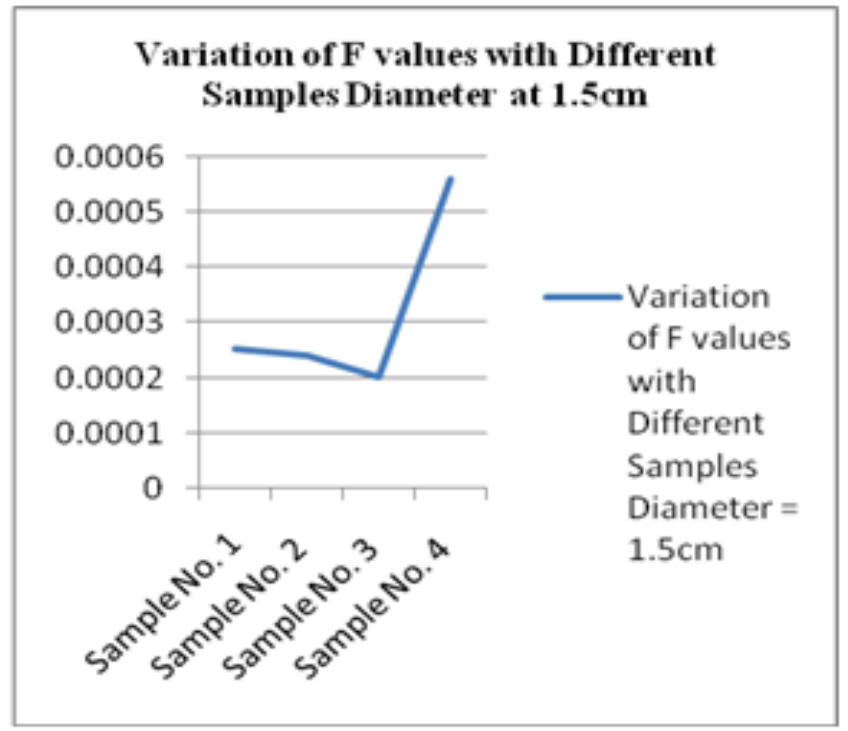

Fig-2 Graph between Samples vs. F values at $2 \mathrm{~cm}$ diameter

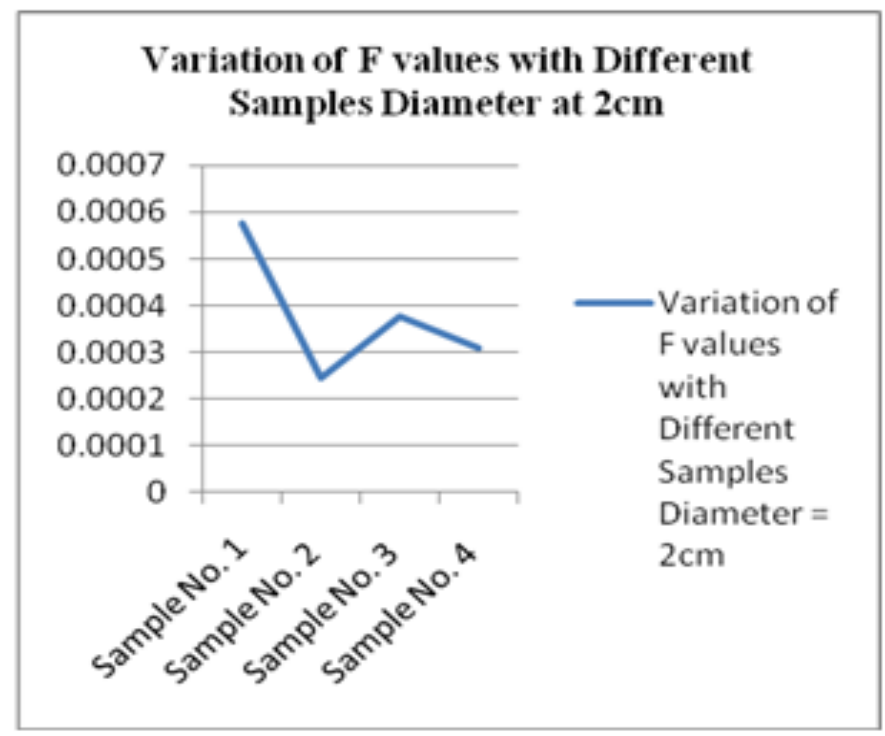

Fig-3 Graph between Samples vs. F values at $1.5 \mathrm{~cm}$ diameter 
Table-6: Physicochemical characteristic of bore well water sample

\begin{tabular}{|l|l|l|l|}
\hline Sr. No. & Parameters & Result & Remark \\
\hline 1 & TDS(ppm) & $101 \mathrm{ppm}$ & Due to turbidity \\
\hline 2 & $\mathrm{pH}$ & 7.42 & Slightly Basic \\
\hline 3 & Conductivity & $0.91 \mathrm{Ms}$ & $\begin{array}{l}\text { Conductivity } \\
\text { due to ions }\end{array}$ \\
\hline 4 & $\begin{array}{l}\text { Total Hardness } \\
(\mathrm{ppm})\end{array}$ & $500 \mathrm{ppm}$ & Hard water \\
\hline 5 & $\begin{array}{l}\text { Temporary } \\
\text { Hardness (ppm) }\end{array}$ & $150 \mathrm{ppm}$ & $\mathrm{CaCO}_{3}$ \\
\hline 6 & $\begin{array}{l}\text { Permanent } \\
\text { Hardness (ppm) }\end{array}$ & $350 \mathrm{ppm}$ & Dissolved Salts \\
\hline 7 & $\begin{array}{l}\text { Dissolves } \\
\text { oxygen (ppm) }\end{array}$ & $7.1 \mathrm{ppm}$ & High \\
\hline 8 & Temperature & 26.10 & Moderate \\
\hline 9 & Humidity & $\begin{array}{l}\text { Min.37\%, } \\
\text { Max. 39\% }\end{array}$ & High \\
\hline 10 & Colour & Colourless & --- \\
\hline 11 & Odour & Odourless & ---- \\
\hline
\end{tabular}

\section{CONCLUSIONS}

Coefficient of pipe friction mostly depends upon the Physico-chemical quality of water which flow through the pipe. From table and graph it is clear that Coefficient of pipe friction initially decreases with the water sample containing 0.5 molar sodium hydroxide solution at 2 diameter pipe but when water containing 0.5 molar sodium hydroxide with 13 gram surfactant it is decreases at same diameter were as water sample containing 0.5 molar sodium hydroxide with 39 gram surfactant it initially decreases then increases in the case of 1.5 diameter pipe.

Coefficient of pipe friction varies with the Physico-chemical feature of water sample. It is lowest with water sample containing 0.5 molar sodium hydroxide.

\section{RESULTS}

Coefficient of pipe friction depends upon the Physicochemical characteristics of water sample and diameter of pipe in which water flow. Addition of suitable quantity of $\mathrm{NaOH}$ and surfactant reduces the coefficient of pipe friction from $5.77 \times 10-3$ to $3.1 \times 10-3$ at diameter 2 and $2.524 \times$ $10-3$ to $2.02 \times 10-3$ at diameter 1.5 but use of excess quantity of surfactant further sudden increases the coefficient of pipe friction from $2.02 \times 10-3$ to $5.61 \times 10-3$. This concept is used in the artificial hydro power generation.

\section{REFERENCES}

[1]. Ir. Peter Romeo Nyarko, (2012), "Heat Load and its Effects on Fluid Friction Factor in Corrugated Pipes" American Journal of Scientific and Industrial Research, 2012, 3(4): 241-251.

[2]. Bruno Brunone, Bryan W. Karney, Michele Mecarelli, and Marco Ferrante (2000), "Velocity Profiles and Unsteady Pipe Friction in Transient Flow" Journal of Water Resources Planning and Management, Vol. 126, No. 4, 236-244
[3]. John F. Ripken and Mem Pilch, (1963), Studies of the Reduction of Pipe Friction with the Non-Newtonian Additive CMC", University Of Minnesota Technical Paper No. 42, Series B

[4]. Osborne Reynolds (1842-1912), "Laminar and Turbulent flows in pipes"

[5]. Gaddamwar, A.G. (2010) "Analytical study of rain water for the determination of polluted or unpolluted zone" International Journal of Environmental Sciences Volume 1, No 6, 2011 pp 1317-1322.

[6]. Lewis F.Moody, Princeton, N.J, (1944), Friction Factor for pipe flow, American society of mechanical engineers [7]. B. J. Mckeon, M. V. Zagarola And A. J. Smits, (2005), "A new friction factor relationship for fully developed pipe flow” J. Fluid Mech. (2005), vol. 538, pp. 429-443

[8]. S. Ghosh, M. Muste, M. Wilson, S. Breczinski, and F. Stern, "Measurement of Flow Rate, Velocity Profile and Friction Factor in Pipe Flows" Mechanics of Fluids and Transfer Processes Exercise Notes for the Pipe Flow TM [9]. M.M.K.Khan (2992) Friction Factor and flow characterisation of non-Newtonian fluids, $11^{\text {th }}$ Australian Fluid mechanics conference University of Tasmania Hobart, Australia

[10]. Ghanbari A., Farshad F. Fred and Rieke H. H. (2011), "Newly developed friction factor correlation for pipe flow and flow assurance" Journal of Chemical Engineering and Materials Science Vol. 2(6), pp. 83-86.

[11]. Gaddamwar A.G., Rajput P.R. (2012) "PhysicoChemical Analysis of Rain Water to Predict Polluted, Unpolluted Regions of Vidharbha and Its Impact on Agricultural Crops" Water Research \& Development, Vol. 2 | No.1\&2 | 54-58.

[12]. Liang Chuan Peng (1989), "Treatment of support friction in pipe stress analysis" The American society of mechanical engineers, PVP-Vol.169 pp 143-148

[13]. Chen, N.H., (1979) "An Explicit Equation for Friction factor in Pipe", Ind. Eng. Chem.Fundam., Vol. 18, No. 3, 296-297,

[14]. Churchill, S.W., (1977) "Friction factor equations spans all fluid-flow ranges.”, Chem.Eng., 91.

[15]. Colebrook, C.F. and White, C.M., (1937) "Experiments with Fluid friction roughened pipes.",Proc. R.Soc.(A), 161.

[16]. Haaland, S.E., (1983) "Simple and Explicit formulas for friction factor in turbulent pipe flow.", Trans. ASME, JFE, 105.

[17]. Moody, L.F., (1944) "Friction factors for pipe flows.", Trans. ASME, 66,641.

[18]. Romeo, E., Royo, C., and Monzon, A., (2002) "Improved explicit equations for estimation of the friction factor in rough and smooth pipes." Chem. Eng. J., 86, 369374.

[19]. Round, G.F., (1980) “An explicit approximation for the friction factor-Reynolds number relation for rough and smooth pipes.”, Can. J. Chem. Eng., 58,122-123.

[20]. Von Bernuth, R. D., and Wilson, T., (1989) "Friction factors for small diameter plastic pipes." J. Hydraul. Eng., 115(2), 183-192.

[21] Wesseling, J., and Homma, F., (1967) "Hydraulic resistance of drain pipes.” Neth. J. Agric. Sci., 15, 183-197. 
[22]. Wood, D.J., (1966) “An Explicit friction factor relationship.”, Civil Eng., 60-61.

[23]. Zagarola, M. V., (1996) "Mean-flow Scaling of Turbulent Pipe Flow,' Ph.D.thesis, Princeton University, USA

\section{BIOGRAPHIES}

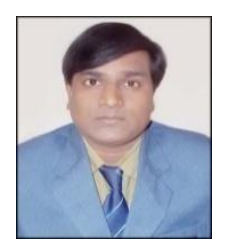

Amrut G.Gaddamwar, Assistant Professor \& HOD Engineering Chemistry, DES's College of engineering \& Technology, Dhamangaon Rly. Qualified UGC-CSIRNET, Published 10 research articles in International journal having good impact factor and Published 07 research articles in National journal having impact factor, Published 06 International Books, Published 01 International Monograph, 12 research articles presented in different national conferences and File one patent.

Email-gamrut_reddy@rediffmail.com

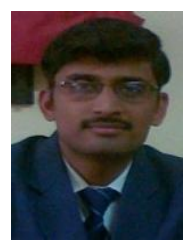

K.R.Banarse, Assistant Professor \& HOD Engineering Physics, DES's College of engineering \& Technology Dhamangaon Rly. Published 07 research articles in International and National journal having good impact factor Attended 02 National STTP

Email-krbanarse@gmail.com

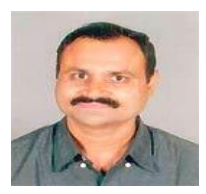

Dr S.P.Yawale, Associate Professor, Department of Physics, Govt. Vidharbha Institute of Science and Humanities, SGBAU Amravati University, Amravati, Maharashtra, India

Dr.P.R.Rajput, Associate professor Department of Chemistry Vidyabharti Science College Amravati

Email-prsrajput@rediffmail.com 